\title{
CARACTERÍSTICAS DA LARANJEIRA ‘VALÊNCIA’ SOBRE CLONES E HÍBRIDOS DE PORTA-ENXERTOS TOLERANTES À TRISTEZA ${ }^{(1)}$
}

\author{
RITA BORDIGNON ${ }^{(2)}$; HERCULANO PENNA MEDINA FILHO ${ }^{(2,5)}$; \\ WALTER JOSÉ SIQUEIRA ${ }^{(3)}$; ROSE MARY PIO ${ }^{(4)}$
}

\begin{abstract}
RESUMO
Estudou-se a variabilidade e o potencial de seleção de 396 híbridos entre os porta-enxertos limão Cravo 'Limeira' (Citrus limonia) (C), tangerina Sunki (C. sunki) (S), laranja Azeda 'São Paulo' (C. aurantium) (A) e Trifoliata 'Davis $\mathrm{A}^{\prime}$ (Poncirus trifoliata) $(\mathrm{T}$ ) tolerantes à tristeza, comparativamente aos genitores Cravo, Sunki e Trifoliata. Foram investigados os híbridos T x A, T x S, S x T, C x S, S x C, C x A e S x A quanto à produção das três primeiras colheitas, produtividade e várias características vegetativas e industriais da laranjeira 'Valência' neles enxertada. Cravo, Trifoliata e os híbridos T x S, S x T, T x A e C x A iniciaram a produção precocemente comparado à Sunki, S x C, C x S e S x A, denotando a dominância da produção precoce do Trifoliata, mesmo nos híbridos com Sunki, e a tendência dos híbridos de Sunki em induzir produção tardia, exceto na combinação com o Trifoliata. A produtividade por área de projeção da copa do Trifoliata foi baixa, ao contrário do Cravo, Sunki e híbridos T x S e S x T. Observou-se boa associação entre os diâmetros dos enxertos e também dos porta-enxertos logo após o plantio no campo e nos quatro anos subseqüentes. Altura, diâmetro da copa, dos troncos do enxerto e porta-enxertos são altamente relacionados e úteis para compor um índice caracterizando o vigor. Trifoliata e Sunki foram os mais divergentes quanto ao vigor e os híbridos entre eles, os que apresentaram maior variabilidade para essa característica. Em 2000, vigor e produção correlacionaram-se com o diâmetro do enxerto em 1996, mostrando ser acertada a prática corrente de se escolherem as mudas mais vigorosas para o plantio comercial, indicando sua utilidade na pré-seleção de mudas de híbridos para o plantio de campos de seleção. A incompatibilidade parcial enxerto/porta-enxerto, observada em 'Valência'/Trifoliata, manifestou-se em seus híbridos, mas não se correlacionou com os atributos produção precoce e elevados ${ }^{\circ}$ Brix e ratio desse genitor, tratando-se de determinantes genéticos independentes. Trifoliata induziu altos valores de ratio do suco e, todos os seus grupos de híbridos foram superiores à Sunki e ao Cravo. Quanto à produção, verificou-se a superioridade do Cravo em relação à Sunki e esta em relação ao Trifoliata, enquanto nos híbridos constatou-se ampla variabilidade genética, sendo 228 significativamente mais produtivos que o Trifoliata, 100 superiores à Sunki e 47 ao Cravo. Os resultados evidenciaram alto potencial de seleção desses híbridos.
\end{abstract}

Palavras-chave: Citrus, Poncirus, melhoramento, seleção, produção, características vegetativas e industriais.

( $\left.{ }^{1}\right)$ Recebido para publicação em 24 de fevereiro de 2003 e aceito em 2 de outubro de 2003.

( ${ }^{2}$ ) Centro de Análise e Pesquisa do Agronegócio do Café Alcides Carvalho, Instituto Agronômico (IAC), Caixa Postal 28, 13001-970 Campinas (SP). E-mail: rita@iac.sp.gov.br

$\left({ }^{3}\right)$ Centro de Pesquisa e Desenvolvimento de Recursos Genéticos Vegetais, Instituto Agronômico (IAC), Campinas (SP).

$\left({ }^{4}\right)$ Centro Avançado de Pesquisa Tecnológica do Agronegócio de Citros Sylvio Moreira, Instituto Agronômico (IAC), Caixa Postal 4, 13490-970 Cordeirópolis (SP).

$\left({ }^{5}\right)$ Com bolsa de produtividade em pesquisa do CNPq. 


\title{
ABSTRACT \\ CHARACTERISTICS OF `VALENCIA' SWEET ORANGE ONTO CLONES AND HYBRID ROOTSTOCKS TOLERANT TO THE TRISTEZA DISEASE
}

\begin{abstract}
Variability and selection potential of 396 hybrids of Rangpur lime 'Limeira', (Citrus limonia) (C), Sunki mandarin (C. sunki) (S), Sour orange 'São Paulo'(C. aurantium) (A) and Trifoliate orange 'Davis $\mathrm{A}^{\prime}$ (Poncirus trifoliata) $(\mathrm{T})$ tolerant to the tristeza disease were studied, comparatively to the genitors Rangpur lime, Sunki and Trifoliate orange. Hybrids TxA, TxS, SxT, CxS, SxC, CxA and SxA were investigated as to yield of first three crops, productivity and several vegetative and industrial characteristics of Valencia sweet orange onto them. Rangpur lime, Trifoliate orange and T $\mathrm{S}, \mathrm{S} \times \mathrm{T}, \mathrm{T} \times \mathrm{A}, \mathrm{C} \times \mathrm{A}$ hybrids initiated yielding before Sunki and S x C, C x S, S x A hybrids. This result indicates a dominance of the precocious yield of Trifoliate even in the hybrids with Sunki and conversely, the opposite trend of Sunki and its hybrids, except in the combination with Trifoliate orange. Yield per canopy area induced by Trifoliate orange was low, contrasting with Rangpur lime, Sunki mandarin and T x S, S x T hybrids. It was observed a close relationship between the diameter of scions, the diameter of rootstocks right after transplant to the field and the same parameters in the subsequent years. Height, canopy, rootstock and scion trunk diameters were highly correlated and useful for composing an index vigor. Trifoliate orange and Sunki mandarin are the most divergent genitors regarding vigor, and the hybrids between them the most variable group. Vigor and yield in 2000 were correlated with scion diameter in 1996, indicating the suitability of customary practice of choosing the most vigorous seedlings for transplanting to commercial fields. Such strategy would be most beneficial if applied for pre-selecting hybrids, before transplant to selection fields. Partial scion/rootstock incompatibility observed in Valencia/ Trifoliate orange was present in Trifoliate hybrids but was not correlated with early yield or industrial attributes of this rootstock. Juice ratio of Trifoliate orange and its hybrids was higher than the ones of Sunki mandarin and Rangpur lime. Yields of Rangpur lime were higher than those of Sunki mandarin and Trifoliate orange. The hybrids displayed considerable genetic variability provided 228 had significant higher yields than Trifoliate orange, 100 higher than Sunki mandarin, and 47 higher than Rangpur lime. The results indicate a good potential selection of such hybrids.
\end{abstract}

Key words: Citrus, Poncirus, breeding, selection, yield, vegetative and industrial characteristics.

\section{INTRODUÇÃO}

Os primeiros trabalhos de melhoramento genético de porta-enxertos, por meio de hibridação e seleção, foram desenvolvidos, de acordo com CAMERON e Frost (1968), por Swingle e Webber, na Flórida, em 1893, realizando milhares de cruzamentos entre as mais diversas espécies de Citrus e gêneros relacionados, produzindo uma imensa variabilidade genética da qual o mundo citrícola até hoje se beneficia. Muitas questões básicas de biologia, genética e melhoramento dos citros foram estudadas de forma intensiva por esses autores e seus seguidores durante mais de 50 anos.

Deu-se grande ênfase à produção de híbridos de copas e de porta-enxertos com subseqüentes testes para tolerância ao frio e à Phytophthora . Paralelamente, na Califórnia, um programa de melhoramento foi também implementado no início do século XX e desenvolvido por várias décadas por H.B. Frost e seus colaboradores. Desses dois programas derivaram muitas das introduções dos Bancos de Germoplasma estabelecidos no Brasil e em vários países.
Elaborados programas de hibridização e testes de porta-enxertos foram também realizados até 1950, em Java, nas Filipinas, no Japão e na Rússia (CAMERON e Frost, 1968).

No Instituto Agronômico (IAC), as pesquisas com porta-enxertos se iniciaram em 1930 e, com o aparecimento da tristeza em 1941, um programa de cooperação com o USDA resultou em uma das mais importantes contribuições científicas para o conhecimento dessa doença no mundo, e também, o início de extensivas avaliações de porta-enxertos, principalmente para as laranjas doces.

A continuidade dessas investigações revelou a excelência do limão Cravo nas nossas condições, bem como as vantagens e deficiências de outros porta-enxertos alternativos como as tangerinas Sunki e Cleópatra, tangelo Orlando, limão Volkameriano entre outros. É consenso geral, refletido pela utilização em larga escala, a preferência dos citricultores pelo limão Cravo, embora existam alternativas bastante promissoras (POMPEU JÚNIOR, 1990; MÜLlER et al., 1996; Figueiredo et al., 1997; TeÓFILO SOBRINHO et al., 1999). O Trifoliata e alguns de seus híbridos têm recebido 
interesse especial, pois, ao induzirem menor porte, possibilitam a redução de espaçamentos de plantio (Phillips, 1969; Teófilo Sobrinho, 1972; Castle, 1987; CAstle et al., 1989; Roose et al., 1989; Ferguson et al., 1990).

Em revisão sobre a qualidade dos frutos, STUCH et al. (1996) compararam a influência de vários porta-enxertos comerciais, incluindo o limão Cravo, a tangerina Sunki, o Trifoliata e a laranja Azeda. Verificaram a qualidade inferior do suco das frutas produzidas sobre Cravo e a melhor qualidade sobre o Trifoliata, sendo intermediária nas plantas sobre Sunki e Azeda. É freqüente na literatura, menções sobre o teor superior de sólidos e melhor coloração dos frutos das plantas em Trifoliata, bem como sua tendência em produzir frutos menores e de maturação tardia.

A tangerina Sunki e a laranja Azeda induzem maturação tardia de frutos com tamanho médio; em limão Cravo, a maturação é intermediária, porém os frutos são geralmente maiores. Essas tendências gerais se observam em regiões distintas como as verificadas por DIEZ e MüLLER (1990) no Uruguai, por FERGUSON et al. (1990) na Califórnia e por CASTLE et al. (1989) na Flórida. Verificaram também tendências quanto às características vegetativas e agronômicas desses porta-enxertos, destacando-se o reduzido vigor das copas em Trifoliata, o oposto em Cravo, Sunki e Azeda e, contrariamente à Sunki, a produção precoce do Cravo e a capacidade do Trifoliata e da Azeda em manterem os frutos nas plantas por prolongados períodos sem deteriorar a qualidade.

No decorrer do programa de melhoramento de porta-enxertos de citros no IAC realizaram-se grande número de polinizações controladas entre clones-elite de porta-enxertos, com o objetivo de se produzir híbridos que associassem características de interesse presentes em clones distintos.

Os híbridos, identificados por eletroforese de isoenzimas e folhas trifolioladas, foram enxertados com borbulhas de laranja 'Valência', transplantados para um campo de observação, estudados quanto a reação à gomose de Phytophthora e, principalmente, a tolerância à tristeza visto a impossibilidade de se produzirem laranjas doces sobre porta-enxertos intolerantes à essa doença, no Brasil.

Neste trabalho, relatam-se os resultados das primeiras avaliações da produção, da produtividade, bem como de algumas características vegetativas e industriais da laranja 'Valência' enxertada em clones genitores e nos híbridos entre eles que se mostraram tolerantes à tristeza. O objetivo dessa investigação foi conhecer o comportamento, a variabilidade e as características gerais desses híbridos tolerantes em relação aos genitores, visando determinar seus potenciais de seleção e, assim, decidir pela continuidade das avaliações por mais alguns anos.

\section{MATERIAL E MÉTODOS}

\subsection{Material vegetal}

Sete diferentes grupos de híbridos (Quadro 1), obtidos por polinizações controladas, identificados por eletroforese de isoenzimas e folhas trifolioladas, enxertados com laranja 'Valência' e transplantados, juntamente com seus genitores para um campo de observação no Centro Avançado de Pesquisa Tecnológica de Agronegócios dos Citros (BordignON et al., 1990; BORDIGNON, 1995; 2000; BALLVE et al., 1991; 1995 ; 1997) foram previamente investigados quanto a reação ao vírus da tristeza (BordiGNON et al., 2004).

Em um total de 128 plantas os genitores, exceto a laranja Azeda, se mostraram tolerantes, bem como 396 dos 544 híbridos entre eles. Esses híbridos e genitores tolerantes, plantados no espaçamento de $8 \times 4 \mathrm{~m}$, foram analisados quanto à produção, à produtividade, às características vegetativas, características relacionadas aos frutos e características industriais da laranjeira 'Valência' sobre eles enxertada, conforme especificados a seguir.

\subsection{Produção}

Refere-se à primeira, segunda e terceira colheitas em 1997, 1998, 1999 e a soma em quilogramas por planta.

\subsection{Características vegetativas}

Obteve-se o diâmetro dos troncos dos portaenxertos em 1996, 1998 e 2000, a partir do perímetro dividido por $\partial$, medido a $3 \mathrm{~cm}$ abaixo do ponto de enxertia. Da mesma forma, obteve-se o diâmetro dos enxertos em 1996 e 2000, a partir do perímetro a 3 $\mathrm{cm}$ acima da enxertia.

Calculou-se o índice expressando o grau de compatibilidade entre enxerto e porta-enxerto, a partir do diâmetro do enxerto em relação ao do porta-enxerto em 2000. Em 1998 e 2000, obteve-se a altura das plantas $(\mathrm{cm})$ com régua graduada, e o diâmetro da copa $(\mathrm{cm})$, a partir da média das larguras das copas medidas, em cada ano, em duas faces ortogonais. Adicionalmente, calculou-se um índice de vigor vegetativo de cada indivíduo, com os valores acima em centímetros, segundo a fórmula:

[altura + diâmetro da copa + 10(diâmetro tronco do porta-enxerto)]/100. 
Quadro 1. Identificação no BAG do CAPTA Citros "Sylvio Moreira", do Instituto Agronômico (IAC), respectivas abreviações e número de plantas dos clones de porta-enxertos e dos seus híbridos tolerantes à tristeza, estudados quanto à produção, às características vegetativas e industriais da laranja 'Valência' sobre eles enxertada

\begin{tabular}{|c|c|c|c|c|}
\hline Espécie & Clone & Identificação no BAG & Abreviatura & $\begin{array}{c}\text { Número } \\
\text { de plantas } \\
\end{array}$ \\
\hline Citrus limonia & Limão Cravo Limeira & $\begin{array}{l}\text { n. }{ }^{\circ} 863 \text { Quadra } 30 \\
\text { Banco de Matrizes } \\
\text { RG } 050 \text { Linha } 14\end{array}$ & $\mathrm{C}$ & 47 \\
\hline Citrus sunki & Tangerina Sunki & $\begin{array}{c}\text { n. } 200 \text { Coleção clones velhos } \\
\text { Banco de Matrizes } \\
\text { RG } 050 \text { Linha } 13\end{array}$ & S & 48 \\
\hline \multirow[t]{2}{*}{ Citrus aurantium } & Laranja Azeda São Paulo & $\begin{array}{c}\text { n. }{ }^{\circ} 244 \text { Coleção clones velhos } \\
\text { n. }{ }^{\circ} 285 \text { Quadra } 16\end{array}$ & & \\
\hline & & RG 050 Linha 12 & A & 0 \\
\hline \multirow[t]{2}{*}{ Poncirus trifoliata } & Trifoliata Davis A & $\begin{array}{l}\text { n. }{ }^{\circ} 848 \text { Quadra } 30 \\
\text { RG } 050 \text { Linha } 4\end{array}$ & $\mathrm{~T}$ & 33 \\
\hline & Híbridos & & & \\
\hline P. trifoliata $\times$ C. aurantium & Trifoliata $\times$ Azeda & RG 050 Linhas 1 e 2 & $\mathrm{~T} \times \mathrm{A}$ & 21 \\
\hline P. trifoliata $\times$ C. sunki & Trifoliata $\times$ Sunki & RG 050 Linha 2 & $\mathrm{~T} \times \mathrm{S}$ & 16 \\
\hline C. sunki $\times$ P. trifoliata & Sunki x Trifoliata & RG 050 Linhas 2 a 4 & $\mathrm{~S} \times \mathrm{T}$ & 45 \\
\hline C. limonia x C. sunki & Cravo x Sunki & RG 050 Linhas 4 e 5 & $C \times S$ & 27 \\
\hline C. sunki x C. limonia & Sunki x Cravo & RG 050 Linhas 5 a 9 & $S \times C$ & 200 \\
\hline C. limonia $\times$ C. aurantium & Cravo x Azeda & RG 050 Linhas 11 e 12 & $\mathrm{C} \times \mathrm{A}$ & 27 \\
\hline C. sunki x C. aurantium & Sunki x Azeda & RG 050 Linhas 9 a 11 & $\mathrm{~S} \times \mathrm{A}$ & 60 \\
\hline Total & & & & 524 \\
\hline
\end{tabular}

\subsection{Produtividade total e em 1999}

Refere-se à produção por área de projeção da copa. Calculou-se a produção total pela soma das produções de 1997, 1998 e 1999. A de 1999 foi também considerada individualmente por ser relativa à maior das três colheitas, portanto, a menos influenciada pela precocidade de início de produção dos diversos materiais. Obteve-se a área de projeção da copa pela fórmula $\partial R^{2}$, em que $R=1 / 2$ do diâmetro da copa, medido em janeiro de 2000.

\subsection{Características relacionadas aos frutos}

Obteve-se a massa média (g) de frutos em 1997, 1998 e 1999, com base em um máximo de dez frutos por planta. A maturação foi subjetivamente estimada por notas de 1 a 6, uma semana antes da colheita, sendo a nota 1 atribuída aos indivíduos que apresentaram a coloração externa dos frutos mais intensa por ter sido verificado melhor coloração interna nesses frutos.

\subsection{Características industriais}

Calculou-se o rendimento de suco em porcentagem, após esmagamento dos frutos em extratora OIC, modelo OTTO 1800, pela relação massa do suco/massa do fruto. O teor de sólidos solúveis totais (SST) foi determinado por leitura direta em refratômetro B\&S, modelo RFM 330, corrigido pela temperatura e acidez e expresso em ${ }^{\circ}$ Brix . A acidez total (AT), indicada em porcentagem, foi obtida por titulação de $25 \mathrm{~mL}$ de suco, com $\mathrm{NaOH} 0,3 \mathrm{~N}$, utilizando-se fenolftaleína como indicador. Com os valores acima, calculou-se a relação sólidos solúveis: acidez (ratio). 


\subsection{Análises estatísticas}

As estatísticas descritivas, médias, desviospadrão, intervalos de confiança da média a 95\% de probabilidade, valores máximos e mínimos, gráficos de distribuição contendo mediana, quartis, valores discrepantes $\left[Q_{3}+1,5\left(Q_{3}-Q_{1}\right)\right.$ e $\left.Q_{1}-1,5\left(Q_{3}-Q_{1}\right)\right]$, bem como correlações de Pearson (método produtomomento), regressões e análises de variância com testes de Kruskall-Wallis foram calculadas através do software estatístico MINITAB versão 13.

A disposição dos genitores e híbridos no campo de observação foi realizada em 14 linhas, com 48 ruas ortogonais, procurando-se deliberadamente, nas linhas, agrupar, à medida do possível, genitores e seus híbridos, de modo que permita comparações e avaliações visuais acuradas, procedimentos extremamente importantes no trabalho de pré-seleção, além de tornar exeqüível e prática a colheita diferenciada e o manejo do campo. Essa estratégia, embora facilite as comparações e o manejo fitotécnico eficiente de um campo extenso $\left(21.504 \mathrm{~m}^{2}\right)$ com centenas de genótipos diferentes é, entretanto, teoricamente limitada em termos ortodoxos da casualização estatística, pois ocorre apenas dentro de cada um dos sete grupos de híbridos e dos três de genitores, porém não entre eles. Não obstante, tal deficiência é passível de ser minimizada pela determinação mais pormenorizada do comportamento das plantas em relação à posição geográfica no campo, fundamento básico da estatística espacial (Duarte, 2000; Vieira, 2000).

Assim, utilizou-se a Regressão Localmente Ponderada (LOWESS) que permite explorar a relação entre duas variáveis sem necessariamente seguir um modelo específico ou assumir uma distribuição normal de valores ou independência do local, e apropriada para avaliar o grau de homogeneidade de um campo, apresentando simetria e contendo grande número de genótipos que, oriundos de combinações gaméticas diferentes, não se constituem em repetições. Desse modo, considerou-se a resposta da produção total e do índice de compatibilidade nas 48 ruas do campo, lembrando que os grupos de genitores e de híbridos estão dispostos nas linhas, portanto, ortogonalmente às ruas onde se localizam os diversos representantes de cada grupo.

Para os pontos da curva da regressão, empregou-se $\mathrm{f}=0,5$ (fração do número total de pontos) e $\mathrm{p}=2$ (número de passos de interações - "robust steps") (Cleveland, 1979). Adicionalmente, aplicou-se o teste não-paramétrico de Mood para as medianas dos valores das 48 ruas do campo (47 graus de liberdade para o Qui-Quadrado), analisando-se os resultados pela amplitude dos respectivos intervalos de confiança a $95 \%$ de probabilidade.

\section{RESULTADOS E DISCUSSÃO}

\subsection{Produção}

As produções totais e parciais da laranja 'Valência' nos três anos indicaram a superioridade do porta-enxerto limão Cravo $\left(x=53,4 \mathrm{~kg}\right.$.planta $\left.{ }^{-1}\right) \mathrm{em}$ relação à Sunki $(\mathrm{x}=41,7)$ e ao Trifoliata $(\mathrm{x}=26,0)$ (Quadro 2, Figura 1). Esses resultados corroboraram amplamente as informações conhecidas de que o Cravo, além de altamente produtivo, induz um início de produção anterior à Sunki, havendo, entretanto, a tendência de Sunki, nos anos subseqüentes, igualar-se ao Cravo. O Trifoliata, sabidamente de produções individuais menores (TeÓFILO SOBRINHO, 1972; AlvarengA, 1986; POMPEU JúNIOR, 1991), mostrou claramente essa característica, apesar de, à semelhança do Cravo, induzir também o início de produção precoce (Figura 1).

Quanto aos sete grupos de híbridos, verificouse que a média de produção de todos eles foi inferior à do Cravo (Figura 1, Quadro 2). É importante salientar que o potencial desses híbridos não pode ser inferido pelo valor de suas médias, como usual em testes de competição, mas sim pelos seus segregantes máximos para essa característica. Ao contrário dos genitores, constituídos de clones nucelares, existe variabilidade genética, não somente entre os grupos de híbridos, mas também dentro de cada grupo, ou seja, cada indivíduo tem um genótipo diferente.

Cabe ressaltar que os valores dos coeficientes de variação dos genitores, que refletem somente a variância ambiental $\left(\delta_{\mathrm{E}}\right)$, se situaram entre $31 \%$ e $37 \%$ enquanto nos híbridos, em que, além da $\delta_{\mathrm{E}}$, tem-se a variância genética, esse coeficiente variou de $34 \%$ a $59 \%$ (Quadro 2). Essa variabilidade e esses segregantes de alta produção podem ser facilmente observados, pois compõem os indivíduos do quarto quartil de cada grupo de híbrido e os valores discrepantes (Figura 1).

Verificou-se existirem, entre os híbridos, 228 deles cuja produção individual foi superior ao limite máximo $(29,5 \mathrm{~kg})$ do intervalo de confiança (IC) da média do Trifoliata, a 95\% de probabilidade; 100 superiores ao IC $(45,8 \mathrm{~kg})$ da Sunki; 47 superiores ao IC $(58,3 \mathrm{~kg})$ do Cravo. Entre esses, verificou-se que sete híbridos (2 T x S, $2 \mathrm{~S} \times \mathrm{T}, 2 \mathrm{~S} \times \mathrm{C}$ e $1 \mathrm{~S} \times \mathrm{A})$ obtiveram produções individuais superiores $(96,7$ a $103,9 \mathrm{~kg})$ à planta de maior produção $(91,6 \mathrm{~kg})$ entre as 47 nucelares do limão Cravo. Os resultados da produção dessas três primeiras colheitas indicam, portanto, um evidente potencial de seleção desses híbridos e sugerem, conseqüentemente, a continuação das colheitas individuais por mais alguns anos. 
Quadro 2. Número de indivíduos (N) e descritores estatísticos da laranja 'Valência' enxertada nos genitores Trifoliata (T), Sunki (S), Cravo (C) e seus híbridos tolerantes à tristeza $(\mathrm{A}=$ genitor intolerante Azeda), avaliados quanto à produção total de frutos, produtividade (produção kg/m² projeção da copa), índice de vigor, peso médio de frutos, ${ }^{\circ}$ Brix e maturação ( $1=$ precoce; $6=$ tardia) nos anos indicados entre parênteses. ICx $=$ intervalo de confiança da média a $95 \%$ de probabilidade; $Q_{1}=$ valor do limite superior do primeiro quartil; $Q_{4}=$ valor do limite inferior do quarto quartil; $C V \%=$ coeficiente de variação

N Média

Desvio-Padrão

ICx Variância

Mínimo

Q1

Produção Total $(1997+1998$ + 1999)

$\begin{array}{rl}32 & 26,0 \\ 48 & 41,7 \\ 47 & 53,4 \\ 20 & 28,6 \\ 60 & 35,4 \\ 27 & 34,5 \\ 16 & 50,1 \\ 45 & 45,4 \\ 200 & 35,9 \\ 26 & 23,6\end{array}$

\section{9,64}

13,91

16,76
17,02

18,85

11,84

28,25

19,69

18,23

13,69

$\mathrm{T}$
$\mathrm{S}$
$\mathrm{C}$

C

S X A

$\mathrm{C} \times \mathrm{A}$

$\mathrm{T} \times \mathrm{S}$

$\mathrm{S} \times \mathrm{T}$

$\mathrm{S} \times \mathrm{C}$
$\mathrm{C} \times \mathrm{S}$

$\mathrm{C} \times \mathrm{S}$

$\mathrm{T}$
$\mathrm{S}$

C

$\mathrm{T} \times \mathrm{A}$

S X A

$\mathrm{C} \times \mathrm{A}$

$\mathrm{T} \times \mathrm{S}$

$\mathrm{S} \times \mathrm{T}$

$\mathrm{S} \times \mathrm{C}$
$\mathrm{C} \times \mathrm{S}$

T

$22,6-29,5$
$37,7-45,8$
$48,5-58,3$
$20,6-36,6$
$30,5-40,2$
$29,8-39,8$
$35,0-65,1$
$39,5-51,3$
$33,4-38,4$
$18,0-29,1$

92,94
193,46

193,46
280,76

280,76
289,78

355,48

140,20

797,90

387,85

332,45
187,32

2,8
15,0
26,0
4,8
0,0
13,8
17,8
20,4
0,0
0,0

Produtividade Total $(1997+1998+1999)$

$\begin{array}{rr}9,6-12,1 & 12,98 \\ 9,9-12,3 & 17,42 \\ 13,8-16,9 & 29,24 \\ 9,4-15,9 & 48,73 \\ 8,3-10,9 & 25,67 \\ 9,8-13,2 & 18,45 \\ 10,6-16,2 & 27,43 \\ 12,6-15,6 & 24,19 \\ 3,9-4,7 & 18,18 \\ 5,1-8,5 & 18,11\end{array}$

Produtividade (1999)

$\begin{array}{rr}6,5-8,3 & 6,18 \\ 7,8-9,8 & 11,62 \\ 8,7-11,1 & 15,76 \\ 6,3-10,8 & 22, \\ 7,2-9,1 & 14,72 \\ 8,1-9,8 & 4,8 \\ 7,4-11,2 & 12,7 \\ 9,1-10,6 & 6,2 \\ 6,6-7,5 & 9, \\ 4,2-6,4 & 7,13\end{array}$

Índice de Vigor (2000)

$6,2-6,8 \quad 0,75$

$8,2-8,6$

0,53

0,73

6,18
11,62
15,76
22,43
14,72
4,85
12,76
6,23
9,57
7,13
igor $(2000)$
0,75
0,53

2,0
3,4
7,0
1,6
0,0
2,6
4,1
6,2
0,0
0,0

Mediana

Q4

Máximo

6,1
6,1
7,1
4,9
5,8
7,6
7,3
7,7
4,9
3,4

20,4
31,7
43,6
17,6
23,3
27,8
27,1
31,4
24,4
13,1

27,7
39,8
52,3
25,1
30,9
31,0
40,6
39,5
31,2
22,8

$\begin{array}{rrr}32,7 & 45,9 & 37,1 \\ 54,4 & 80,2 & 33,3 \\ 62,8 & 91,6 & 31,4 \\ 36,4 & 78,1 & 59,5 \\ 50,1 & 96,9 & 53,2 \\ 41,0 & 65,2 & 34,3 \\ 76,9 & 103,9 & 56,4 \\ 55,8 & 100,6 & 43,4 \\ 47,2 & 96,7 & 50,8 \\ 29,3 & 51,9 & 58,0\end{array}$

$\begin{array}{rrrrr}8,5 & 10,8 & 13,1 & 19,1 & 33,0 \\ 7,9 & 10,9 & 13,1 & 25,5 & 37,6 \\ 11,5 & 15,0 & 18,9 & 38,7 & 35,3 \\ 7,8 & 12,8 & 17,9 & 26,4 & 55,5 \\ 6,3 & 8,4 & 12,7 & 23,6 & 52,8 \\ 8,3 & 10,8 & 13,9 & 22,2 & 37,3 \\ 9,3 & 13,3 & 18,0 & 21,2 & 39,1 \\ 10,1 & 13,0 & 18,0 & 24,2 & 34,9 \\ 5,2 & 7,5 & 10,9 & 25,8 & 51,6 \\ 4,0 & 5,7 & 9,6 & 18,3 & 62,5\end{array}$

$\begin{array}{rrrr}7,5 & 8,8 & 11,2 & 33,6 \\ 8,5 & 9,9 & 17,2 & 38,7 \\ 8,7 & 13,2 & 23,9 & 40,1 \\ 8,7 & 10,3 & 18,3 & 55,1 \\ 8,1 & 10,5 & 19,7 & 46,8 \\ 8,9 & 10,4 & 13,0 & 24,7 \\ 8,7 & 12,3 & 15,9 & 38,4 \\ 10,2 & 11,3 & 16,1 & 25,1 \\ 6,9 & 9,0 & 16,2 & 44,1 \\ 5,4 & 7,4 & 9,8 & 50,4 \\ & & & \\ 6,5 & & & \\ 8,5 & 7,2 & 7,9 & 13,2 \\ & 8,8 & 10,1 & 8,7\end{array}$




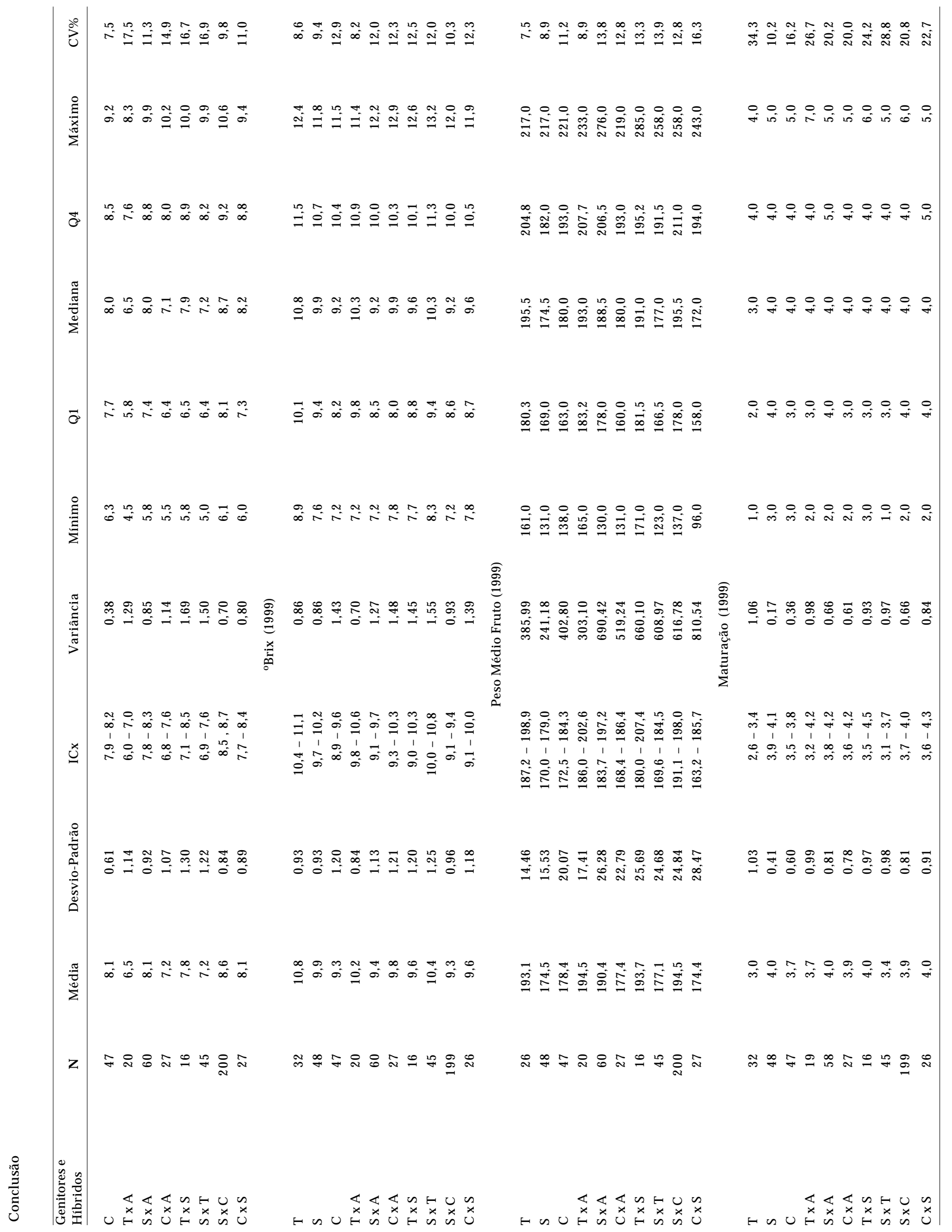



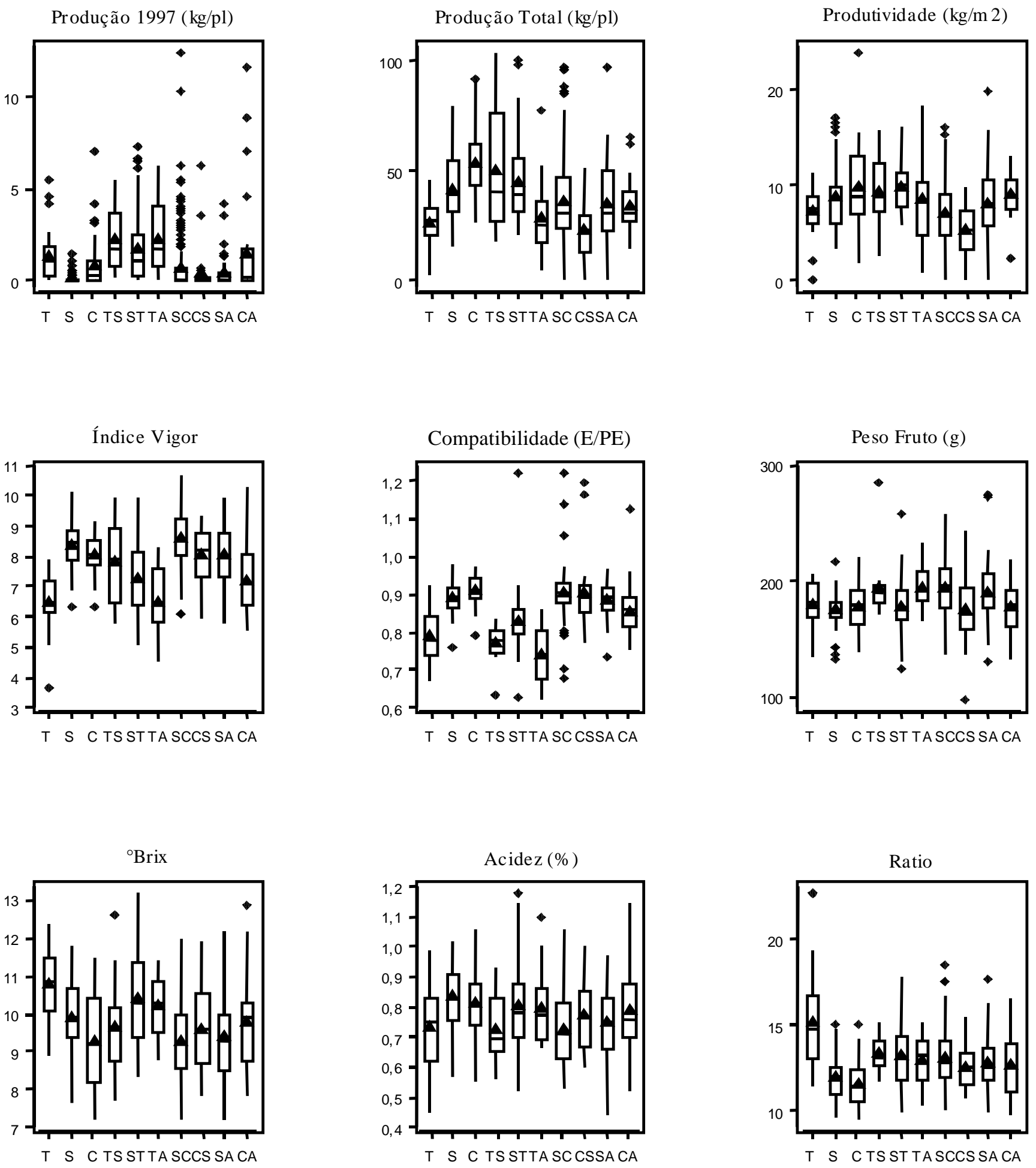

Figura 1. Distribuição dos valores de características produtivas, vegetativas e industriais da laranja 'Valência' enxertada nos genitores Trifoliata $(\mathrm{T})$, Sunki $(\mathrm{S})$, Cravo $(\mathrm{C})$, e seus híbridos tolerantes à tristeza (A = genitor intolerante Azeda). Testes de Kruskall-Wallis, p<0,05. 
Como se observa na figura 1, em relação às produções de 1997 e à soma de 1997, 1998 e 1999, é evidente o início de produção precoce, não somente do Trifoliata, mas também do Cravo em relação à Sunki, confirmando as informações conhecidas. Entre os híbridos, é notória a precocidade das combinações Trifoliata x Sunki, Sunki x Trifoliata, Trifoliata x Azeda e Cravo x Azeda e o início tardio de produção das combinações Sunki x Cravo, Cravo x Sunki e Sunki x Azeda, denotando a influência e a dominância da produção precoce do Trifoliata, mesmo em combinação com a Sunki, e a tendência de híbridos da Sunki em induzir produções tardias, exceto na combinação com o Trifoliata.

Nas últimas décadas, tem-se conceituado que, apesar da baixa produção do Trifoliata, sua utilização comercial como porta-enxerto de laranjas doces pode ser vantajosa desde que utilizados menores espaçamentos, viabilizados pelo menor vigor das plantas nele enxertadas. No geral, tais estudos salientam boa produtividade do Trifoliata quando se considera sua produção por $\mathrm{m}^{3}$ de copa (TEófilo SoBRINHO, 1972). Entretanto, a produção por metro quadrado da projeção da copa das plantas é também bastante pertinente para se avaliar a produtividade, visto ser o diâmetro da copa um parâmetro fidedigno que determina o espaçamento mínimo entre as plantas na rua e, conseqüentemente, o limite máximo de densidade de plantio.

Verificou-se que a produção por metro quadrado de projeção da copa do Trifoliata, ao contrário do que se esperava, não é mais alta que a da Sunki ou Cravo, sendo esse último o de maior produtividade, considerando-se tanto a produção acumulada dos três primeiros anos como também quando considerada apenas a de 1999, a qual estaria estimando esse índice com uma menor influência da característica precocidade de produção (Figura 1; Quadro 2). O mesmo foi observado por AlvaRENGa (1986) estudando a laranja 'Valência' enxertada, entre outros, em Cravo e Trifoliata.

Considerando a produtividade e a produção total, verifica-se existir uma tendência dos grupos de maior produção serem também os de maior produtividade por área, como Cravo, híbridos Trifoliata $\mathrm{x}$ Sunki e Sunki x Trifoliata (Figura 1). Nota-se também que a produtividade independe do vigor, visto serem Sunki e seus híbridos com Cravo, os mais vigorosos, porém, relativamente, de baixa produtividade.

\subsection{Características vegetativas}

Os valores dos diâmetros dos porta-enxertos em 1996 (Quadro 3), logo após o transplante das plantas para o campo, evidenciaram o menor desenvolvimento inicial do Trifoliata $(x=1,5 \mathrm{~cm})$ e o maior vigor da Sunki $(x=2,3)$, do Cravo $(x=2,6)$ e dos híbridos $C x$ $S(x=2,9)$ e $S$ x C $(x=2,5)$. Observou-se boa relação entre o diâmetro inicial em 1996 dos porta-enxertos e esse parâmetro nos anos de 1998 e 2000 com médias de correlações significativas $(p \leq 0,05)$ de $36 \%$ a $86 \%$ entre as três avaliações, calculadas para os genitores e seus híbridos (Quadro 4). Quanto ao diâmetro do tronco do enxerto em 1996 e 2000, verifica-se pelo quadro 3, que os maiores valores foram observados nas plantas sobre Cravo, Sunki e híbridos entre eles, e os menores, no Trifoliata e seus híbridos. Em 2000, o diâmetro se mostrou correlacionado $(x=47 \%)$ a esse parâmetro em 1996 (4 x 5), evidenciado por seis correlações significativas $(\mathrm{p} \leq 0,05)$ entre 10 calculadas (Quadro 4). O diâmetro da copa em 1998 (Quadro 3) foi altamente relacionado $(70 \%, \mathrm{p} \leq 0,05)$ com o diâmetro medido em 2000. Da mesma forma, são as alturas ( $71 \%$ ) e índices de vigor $(80 \%)$, verificando-se dez correlações significativas $(p \leq 0,05)$ das dez testadas para as três características $(6 \times 7,8 \times 9,10 \times 11)$ (Quadro 4).

A altura das plantas, diâmetro da copa e diâmetros do tronco do porta-enxerto e do enxerto (Quadro 3) são características altamente correlacionadas $(60 \%$ a $81 \%)$ nas medições de 1998 e 2000. Das 40 correlações testadas, 39 foram significativas (Quadro 4). O índice de vigor, ao considerar altura das plantas, diâmetro da copa e do tronco do porta-enxerto, apesar da evidente correlação de seus componentes, é possivelmente o parâmetro que melhor reflete o vigor vegetativo das plantas. Observando a figura 1 e os valores dos quadros 2 e 3 , verifica-se o alto vigor vegetativo da Sunki $(x=8,4)$, Cravo $(x=$ $8,1)$, híbridos entre eles $(x=8,6$ e 8,1) e dos híbridos Sunki x Azeda $(x=8,1)$, contrastando com os reduzidos valores do Trifoliata $(x=6,5)$ e seus híbridos com Azeda $(x=6,5)$. Nota-se pela figura 1 , a maior variabilidade para essa característica observada entre os híbridos recíprocos de Trifoliata $x$ Sunki, lembrando serem Trifoliata e Sunki os genitores mais divergentes para essa característica. Essa ampla variabilidade observada nos híbridos resultante do cruzamento entre dois genótipos (clones genitores) é, por certo, uma indicação que este parâmetro está sob o controle genético de vários loci heterozigotos.

Como esperado, os índices de vigor obtidos em 2000 são altamente correlacionados $(x=80 \%, p \leq 0,05)$ com os obtidos em 1998 em todas as 10 associações testadas (Quadro 4; correlação $10 \times 11$ ), mostrando que essa característica não se modificou no período analisado. $\mathrm{O}$ vigor das plantas em 2000 se correlaciona com o diâmetro do enxerto em 1996 com maior freqüência $(5 \mathrm{em} 7)$ e maior intensidade $(\mathrm{x}=45 \%)$ entre os híbridos do que entre os genitores ( $2 \mathrm{em} 3, \mathrm{x}=35 \%$ ) (Quadro 4; correlação 4 x 11). 
Quadro 3. Médias das características vegetativas (1 a 9) em cm, $\varnothing \mathrm{PE}=$ diâmetro do tronco do porta-enxerto, $\varnothing \mathrm{E}=$ diâmetro do tronco do enxerto, índice de vigor (10 e 11) e respectivos anos de obtenção, produção total de frutos em kg (12) refere-se às colheitas de 1997+1998+1999 e, produtividade por área (13) à produção total em kg/área da projeção da copa em $\mathrm{m}^{2}$ em 2000, T, S, C e A referem-se respectivamente aos porta-enxertos Trifoliata, Sunki, Cravo e Azeda e correspondentes híbridos

\begin{tabular}{|c|c|c|c|c|c|c|c|c|c|c|c|c|c|}
\hline $\begin{array}{c}\text { Genitores e } \\
\text { Híbridos }\end{array}$ & $\begin{array}{c}1 \\
\varnothing \mathrm{PE} \\
1996 \\
\end{array}$ & $\begin{array}{c}2 \\
\varnothing \mathrm{PE} \\
1998 \\
\end{array}$ & $\begin{array}{c}3 \\
\varnothing \mathrm{PE} \\
2000 \\
\end{array}$ & $\begin{array}{c}4 \\
\varnothing \mathrm{E} \\
1996 \\
\end{array}$ & $\begin{array}{c}5 \\
\varnothing \mathrm{E} \\
2000 \\
\end{array}$ & $\begin{array}{c}6 \\
\varnothing \mathrm{COPA} \\
1998 \\
\end{array}$ & $\begin{array}{c}7 \\
\varnothing \mathrm{COPA} \\
2000 \\
\end{array}$ & $\begin{array}{c}8 \\
\text { ALTURA } \\
1998 \\
\end{array}$ & $\begin{array}{c}9 \\
\text { ALTURA } \\
2000\end{array}$ & $\begin{array}{c}10 \\
\text { IV } \\
1998 \\
\end{array}$ & $\begin{array}{c}11 \\
\text { IV } \\
2000 \\
\end{array}$ & $\begin{array}{c}12 \\
\text { PROD. } \\
\text { TOTAL }\end{array}$ & $\begin{array}{c}13 \\
\text { PROD. } \\
\text { ÁREA }\end{array}$ \\
\hline $\mathrm{T}$ & 1,5 & 7,3 & 30,1 & 0,9 & 23,6 & 106,7 & 173,4 & 148,9 & 174,4 & 3,3 & 6,5 & 26,0 & 10,9 \\
\hline$S$ & 2,3 & 9,2 & 38,7 & 1,7 & 34,6 & 149,0 & 221,0 & 207,7 & 232,3 & 4,5 & 8,4 & 41,8 & 11,1 \\
\hline$C$ & 2,6 & 9,4 & 37,1 & 2,0 & 33,8 & 144,1 & 211,9 & 209,5 & 222,7 & 4,5 & 8,1 & 53,4 & 15,3 \\
\hline$T \times S$ & 2,4 & 9,0 & 34,9 & 1,5 & 27,0 & 141,6 & 218,0 & 195,0 & 213,7 & 4,3 & 7,8 & 50,1 & 13,4 \\
\hline$S \times T$ & 2,4 & 8,4 & 32,5 & 1,6 & 26,8 & 136,0 & 203,0 & 185,7 & 196,3 & 4,1 & 7,2 & 45,4 & 14,1 \\
\hline$S \times C$ & 2,5 & 9,0 & 38,4 & 1,9 & 34,7 & 147,6 & 237,0 & 203,3 & 238,6 & 4,4 & 8,6 & 35,9 & 8,2 \\
\hline$S \times A$ & 2,2 & 8,6 & 36,2 & 1,6 & 32,1 & 141,5 & 217,1 & 199,7 & 229,6 & 4,3 & 8,1 & 35,4 & 9,6 \\
\hline$C \times A$ & 2,1 & 8,0 & 32,3 & 1,7 & 27,6 & 134,6 & 199,0 & 182,8 & 200,7 & 4,0 & 7,2 & 34,5 & 11,5 \\
\hline $\mathrm{T} \times \mathrm{A}$ & 2,3 & 7,4 & 29,3 & 1,5 & 21,6 & 126,2 & 175,6 & 169,0 & 181,0 & 3,7 & 6,5 & 28,6 & 12,6 \\
\hline$C \times S$ & 2,9 & 8,5 & 36,5 & 1,9 & 33,0 & 135,2 & 213,1 & 200,0 & 228,7 & 4,2 & 8,1 & 23,6 & 6,8 \\
\hline
\end{tabular}

Quadro 4. Valores em porcentagem das correlações (Pearson, produto-momento) significativas ( $\mathrm{p}<0,05)$ entre alguns parâmetros $(1$ a 13$)$ do Quadro 3 calculados para genitores e híbridos de porta-enxertos, $\mathrm{N}=$ número de correlações significativas entre as dez testadas e $\mathrm{X}=$ média dos valores das correlações significativas

\begin{tabular}{|c|c|c|c|c|c|c|c|c|c|c|c|c|c|c|}
\hline $\begin{array}{c}\text { Genitores e } \\
\text { Híbridos }\end{array}$ & $1 \times 2$ & $1 \times 3$ & $2 \times 3$ & $4 \times 5$ & $6 \times 7$ & $8 \times 9$ & $10 \times 11$ & $4 \times 11$ & $6 \times 8$ & $7 \times 9$ & $2 \times 8$ & $5 \times 9$ & $4 \times 12$ & $4 \times 13$ \\
\hline $\mathrm{T}$ & Ns & ns & 75 & ns & 75 & 85 & 87 & ns & 62 & 82 & 67 & 72 & 43 & ns \\
\hline$S$ & 36 & ns & 76 & 38 & 77 & 77 & 84 & 33 & 59 & 81 & 58 & 82 & ns & ns \\
\hline C & 57 & 44 & 74 & 48 & 70 & 54 & 75 & 38 & 40 & 73 & 73 & 65 & 37 & ns \\
\hline $\mathrm{T} \times \mathrm{S}$ & 74 & 66 & 81 & 72 & 52 & 85 & 88 & 69 & 83 & ns & 90 & 95 & 64 & ns \\
\hline $\mathrm{S} \times \mathrm{T}$ & 45 & ns & 74 & ns & 76 & 76 & 85 & 36 & 57 & 87 & 54 & 77 & 55 & ns \\
\hline$S \times C$ & 42 & 37 & 68 & 33 & 74 & 58 & 80 & 31 & 55 & 81 & 62 & 71 & 28 & ns \\
\hline$S \times A$ & 52 & 49 & 70 & 40 & 81 & 58 & 83 & 37 & 61 & 77 & 62 & 75 & 47 & ns \\
\hline$C \times A$ & 50 & ns & 78 & ns & 75 & 81 & 81 & ns & 69 & 83 & 68 & 79 & 40 & ns \\
\hline $\mathrm{T} \times \mathrm{A}$ & 51 & 50 & 86 & 48 & 53 & 69 & 74 & 54 & 51 & 85 & 76 & 83 & 48 & ns \\
\hline$C \times S$ & 44 & ns & ns & ns & 64 & 70 & 64 & ns & 59 & 83 & 50 & 65 & 55 & ns \\
\hline $\mathrm{N} / 10$ & $9 / 10$ & $5 / 10$ & $9 / 10$ & $6 / 10$ & $10 / 10$ & $10 / 10$ & $10 / 10$ & $7 / 10$ & $10 / 10$ & $9 / 10$ & $10 / 10$ & $10 / 10$ & $9 / 10$ & $0 / 10$ \\
\hline$x$ & 50 & 49 & 76 & 47 & 70 & 71 & 80 & 43 & 60 & 81 & 66 & 76 & 46 & _ \\
\hline
\end{tabular}


Esses resultados atestam o acerto da prática corrente de se escolherem as mudas mais vigorosas para plantios comerciais e indicam ser um procedimento que traria benefício ainda maior no estabelecimento de campos de seleção onde existe variabilidade genética entre as plantas. Tal seleção precoce seria extremamente eficiente para reduzir o número de híbridos a serem estudados, respeitadas, entretanto, as diversas características dos genitores e o interesse da seleção para plantas com maior ou menor vigor vegetativo. A seleção precoce de plantas apresentando enxertos vigorosos seria também interessante, a fim de enriquecer a população de híbridos a serem posteriormente avaliados para produção pois, em todos os sete grupos de híbridos estudados foram significativas $(\mathrm{p} \leq 0,05)$ e de intensidade razoável as correlações (Quadro 4; correlações $4 \times 12$ ) do diâmetro do enxerto em 1996 com a produção total ( $\mathrm{x}=48 \%$ ). Não existe, porém, relação com a produtividade (Quadro 4; correlações $4 \times 13$ ).

É conhecida a reação de incompatibilidade que se manifesta em algumas combinações de laranjas doces enxertadas sobre Trifoliata (WEBBER, 1948). Ao contrário das totalmente compatíveis, nas quais enxerto e porta-enxerto têm desenvolvimento semelhante, aquelas com reação de incompatibilidade exibem tipicamente menor desenvolvimento do tronco da copa. No Trifoliata, a relação média entre os diâmetros dos troncos do enxerto/porta-enxerto foi de $0,79 \pm 0,063$ enquanto em Sunki e Cravo foi de $0,89 \pm$ 0,044 e $0,91 \pm 0,039$ respectivamente. Observou-se que essa característica do Trifoliata também se manifesta em todos os seus híbridos e, com maior intensidade, naqueles grupos em que é o genitor feminino como Trifoliata $x$ Sunki $(x=0,77 \pm 0,050)$ e Trifoliata $x$ Azeda $(x=0,74 \pm 0,067)$ comparados com Sunki $x$ Trifoliata $(x=0,83 \pm 0,080)$. Nesses híbridos de Trifoliata com Sunki e Azeda investigaram-se possíveis associações que essa característica do Trifoliata pudesse ter com seus atributos como precocidade de produção, Brix elevado e coloração externa intensa dos frutos. Nenhuma das nove associações testadas resultou em valores de correlações significativas, mostrando que essa característica, facilmente identificável, não se constitui em um critério indireto de seleção precoce de híbridos de Trifoliata e indica serem características genéticas independentes.

\subsection{Características relacionadas aos frutos}

Massa média - As massas médias de frutos observadas nas colheitas de 1997, 1998 e 1999 não mostraram nenhuma tendência marcante dos genitores ou híbridos, verificando-se, nos três anos, uma alternância de seus valores relativos. As diferenças notadas indicam o Trifoliata ter produzido frutos menores nas duas primeiras colheitas ( $\mathrm{x}=188$ e $210 \mathrm{~g})$ quando comparados com os da Sunki (209 e 213 g) e Cravo (214 e $220 \mathrm{~g}$ ). Sabe-se que o tamanho dos frutos apresenta sensíveis variações de ano para ano nos pomares comerciais e se relaciona com idade da planta, condições climáticas, nutrição, nível de produção, não raro interagindo e antagonizando seus efeitos.

No presente caso, verificou-se que o tamanho dos frutos foi bastante diferente nos três anos. Enquanto em 1997 (primeira produção), a média de 278 plantas, compreendendo os dez diferentes grupos estudados, foi de $205 \pm 38 \mathrm{~g}$, em 1998 (479 plantas), 217 $\pm 24 \mathrm{~g}$ e, em 1999 (521 plantas), $183 \pm 23 \mathrm{~g}$. Os frutos dessa última colheita podem ter sido menores devido tanto às condições climáticas desfavoráveis como ao fato de as plantas terem aumentado substancialmente a produção por ser esta última já a terceira colheita. As maiores variações na massa média dos frutos dos genitores e híbridos ocorreram na colheita de 1998, a de maior massa média, e a de menor variação, na colheita de 1999 (Figura 1), com a menor massa média.

Maturação, Brix, acidez, ratio, rendimento As considerações a respeito da influência dos genitores e híbridos nesses parâmetros da laranja 'Valência' (Quadro 2; Figura 1) são bastante limitadas pelo fato de terem sido realizadas apenas no terceiro ano e em uma única ocasião. Sabe-se que essas características apresentam variações de ano para ano e fazem parte de um processo fisiológico dinâmico que evolui no decorrer do período da safra. Gradativamente, a coloração da casca se intensifica, a acidez decresce enquanto o Brix e o ratio aumentam (Di Giorgi et al., 1993; Domingues et al., 1996). São portanto, mais bem avaliados por estudo dinâmico durante todo o período de duração do processo.

A avaliação em apenas uma ocasião poderá refletir as diferenças nos valores finais de Brix, acidez e ratio, mas, alternativamente essas diferenças poderiam também ser reflexo de diferentes estádios de maturação. O Trifoliata é conhecido como um portaenxerto que induz frutos menores, de altos Brix e ratio, boa coloração e maturação tardia (STUCHI et al., 1996). As avaliações visuais da maturação (Quadro 2) mostraram que 'Valência' enxertada no Trifoliata é mais precoce que as enxertadas em Sunki, Cravo e híbridos porém esse fato pode ser apenas devido à melhor coloração externa dos frutos, critério utilizado para avaliação. Entretanto, cabe salientar que o Trifoliata induziu também maiores valores de Brix diminuição na acidez e altos valores de ratio, características indicativas de maior precocidade e qualidade dos frutos (Figura 1; Quadro 2). 
É possível que avaliações no período ideal de maturação venham mostrar que o Trifoliata pode induzir diferenças ainda maiores. Dentre todos os híbridos, os que mais se aproximaram dessas características na época da avaliação foram os híbridos Sunki x Trifoliata. A Tangerina Sunki, conhecida por induzir às copas maturação tardia e qualidade superior ao Cravo, assim se comportou na avaliação visual e nos valores de Brix e acidez. Com referência ao ratio, é interessante notar a superioridade do Trifoliata e de todos os híbridos em comparação com Sunki e Cravo (Figura 1). Quanto ao rendimento de suco, as variações observadas não foram significativas.

Controle local. Verificou-se que o campo é razoavelmente homogêneo, pela localização das plantas mais produtivas ao nível de $25 \%$ e $5 \%$ de cada genitor e de cada grupo de híbridos (Figura 2A). Parece existir uma leve tendência para a ocorrência de maior número de plantas mais produtivas dos dois níveis considerados, nas últimas ruas em comparação com as primeiras. Na figura $2 \mathrm{~B}$, quantitativamente, esse fato se verifica quando são consideradas todas as plantas e também somente as $25 \%$ mais produtivas, pela análise de regressão localmente ponderada. Embora essa tendência seja evidente, sua magnitude, entretanto, é pequena. De fato, o teste de Mood, embora significativo $(p=0,235)$, revelou que, das 48 ruas testadas, apenas uma (rua 42) tem a mediana significativamente diferente a $95 \%$ de probabilidade.

A compatibilidade entre enxertos e porta-enxertos, refletida pela relação entre seus diâmetros, é uma característica inerente apenas aos genótipos das plantas, não sendo esperado uma influencia da posição no campo, desde que, dentro de cada grupo nas linhas, as plantas estivessem ao acaso e, nas ruas, os grupos estivessem representados em disposição aproximadamente simétrica. Conforme se observa na figura $2 \mathrm{C}$, os valores para essa característica são totalmente independentes da posição das plantas no campo, da produção e, consequentemente, casualizados dentro de cada grupo de híbridos e de genitores.

Esses resultados indicam, portanto, ser a área experimental razoavelmente homogênea e que não existe necessidade de, a posteriori, estratificar o campo para futura seleção dos híbridos ou determinar seus potenciais, desde que se adote uma intensidade de seleção moderada.

\section{CONCLUSÕES}

1. Foi marcante o início precoce de produção e a dominância dessa característica do Trifoliata em seus híbridos, mesmo em combinação com a Sunki.
Sunki e seus híbridos, exceto nas combinações com o Trifoliata, induzem início de produções tardias.

2. Produtividade por metro quadrado da projeção da copa foi máxima no Cravo, seguida da Sunki e menor no Trifoliata. Maior produção relacionou-se à maior produtividade por área da projeção da copa em Cravo e híbridos recíprocos T x S. Essa característica não se relacionou ao vigor.

3. O diâmetro inicial (1996) dos porta-enxertos relacionou-se, nos genitores e híbridos, significativamente entre $45 \%$ e $82 \%$ com esse mesmo parâmetro em 1998 e 2000 e com a produção total (48\%). O diâmetro do enxerto em 2000 correlacionouse significativamente com as avaliações de 1996 (49\%). A altura das plantas, o diâmetro da copa e o do tronco foram altamente correlacionadas e úteis para compor o índice de vigor que, em 2000, correlacionou-se com o diâmetro do enxerto em 1996, com maior freqüência e intensidade entre os híbridos que entre os genitores. Tais correlações atestam o mérito da prática corrente de se escolherem as mudas mais vigorosas para plantios comerciais e indicam ter esse procedimento, grande eficiência no estabelecimento de campos visando à seleção de híbridos vigorosos e de elevada produção.

4. O maior vigor vegetativo foi observado nas plantas enxertadas em Sunki, Cravo e híbridos entre eles, sendo os menores nas plantas em Trifoliata e seus híbridos com Azeda. A incompatibilidade parcial das plantas enxertadas em Trifoliata é dominante, sendo observada em seus híbridos. Neles, a reação de incompatibilidade não se correlacionou com precocidade do início de produção, Brix elevado ou maturação precoce, atributos do Trifoliata sendo, portanto, características geneticamente independentes.

5. Os valores de Brix, acidez e ratio corroboraram a conhecida superioridade do Trifoliata. Da mesma forma, comportaram-se todos seus grupos de híbridos em relação a Sunki e a Cravo.

6. Entre os 396 híbridos tolerantes à tristeza, 228 foram mais produtivos que o Trifoliata, 100 mais que a Sunki e 47 mais que o Cravo, considerando os limites superiores do Intervalo de Confiança da média a $95 \%$ de probabilidade. Os grupos que apresentaram maiores proporções de híbridos superiores foram os de Trifoliata $x$ Sunki e seu recíproco.

7. Os resultados mostraram ampla variabilidade genética entre os híbridos para a maioria das características avaliadas, principalmente para produção, indicando existir grande potencial de seleção de porta-enxertos. 

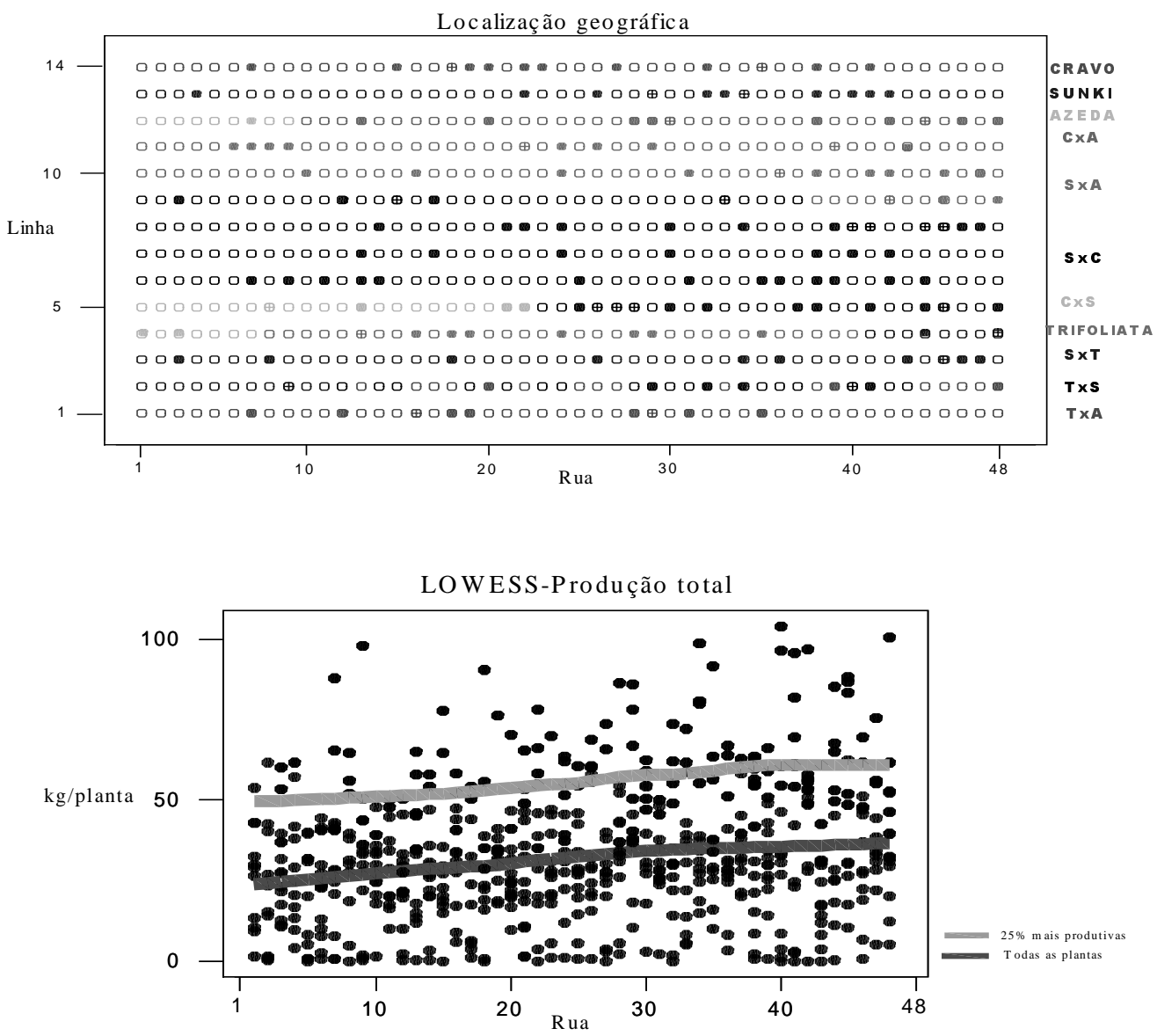

LOWESS-Compatibilidade enxerto/porta-enxerto

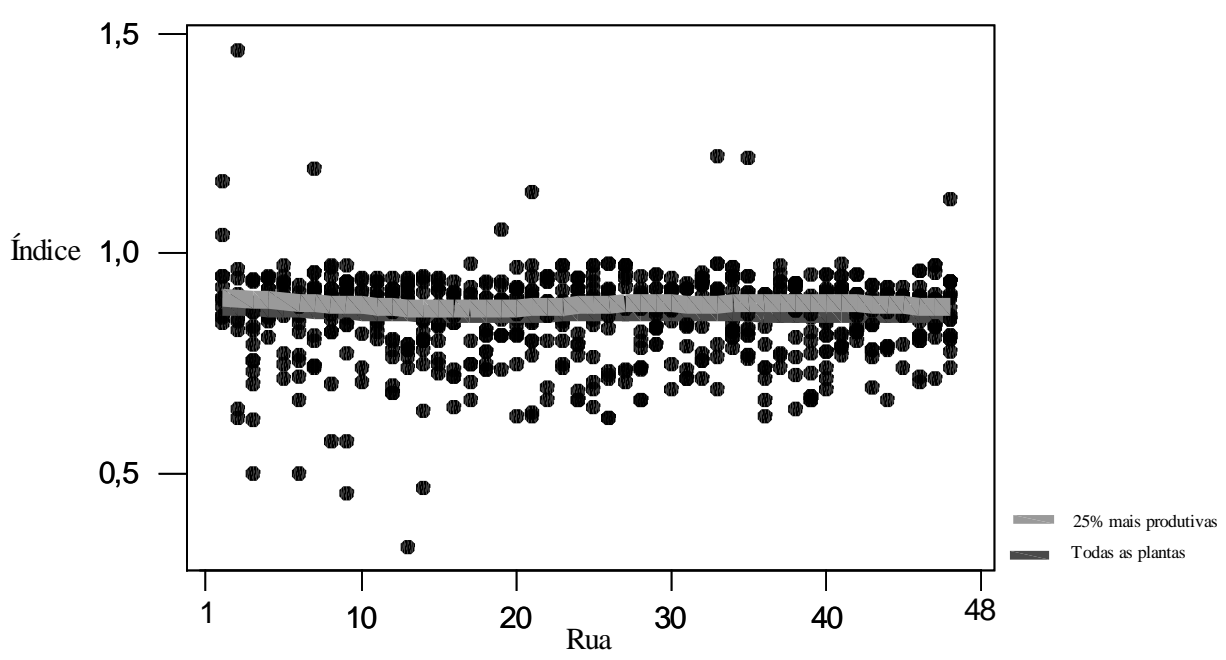

Figura 2. Localização geográfica das linhas e ruas do campo de observação com os genitores Cravo (C), Sunki (S), Azeda (A), Trifoliata (T) e híbridos entre eles, indicando a localização das plantas $25 \%(\bullet)$ e $5 \%(\oplus)$ mais produtivas. Regressão localmente ponderada (LOWESS) das produções de todas as plantas e também das $25 \%$ mais produtivas dos genitores e de cada grupo de híbridos, conforme a localização geográfica nas diversas ruas do campo. LOWESS relativa ao índice de compatibilidade enxerto/porta-enxerto de todas as plantas e das $25 \%$ mais produtivas. 


\section{AGRADECIMENTOS}

Os autores agradecem aos pesquisadores Jorgino Pompeu Júnior e Joaquim Teófilo Sobrinho, do Centro Avançado de Pesquisa Tecnológica do Agronegócio de Citros, pelas sugestões durante o desenvolvimento da pesquisa, a Debir N. Gomes pela condução do campo, a Valéria X. P. Garcia pelas análises industriais e a Silvia Luisa S. Lima pela digitação.

\section{REFERÊNCIAS BIBLIOGRÁFICAS}

ALVARENGA, L.R.; BENDEZU, J.M.; TEIXEIRA, S.L.; GAMA, A.M.P. Comportamento da laranjeira Valência (Citrus sinensis $(\mathrm{L}$,$) Osb,) sobre 12$ porta-enxertos em Porteirinha - MG, In: CONGRESSO BRASILEIRO DE FRUTICULTURA, 8., 1986, Brasília, Anais... Brasília: Sociedade Brasileira de Fruticultura, 1986. v.1, p.153-159.

BALLVÉ, R.M.L.; BORDIGNON, R.; MEDINA FILHO, H.P.; SIQUEIRA, W.J.; TEÓFILO SOBRINHO, J.; POMPEU JUNIOR, J. Isoenzimas na identificação precoce de híbridos e clones nucelares no melhoramento de citros. Bragantia, Campinas, v.50, n.1, p.57-76, 1991.

BALLVÉ, R.M.L.; MEDINA FILHO, H.P.; BORDIGNON, R. Identification of reciprocal hybrids in citrus by the broadness of leaf petiole wing. Brazilian Journal of Genetics, Ribeirão Preto, v.20, n.4, p.697-702, 1997.

BALLVÉ, R.M.L.; MEDINA FILHO, H.P.; BORDIGNON, R.; LIMA, M.M.A. Methodology for starch gel electrophoresis and protocols for isozyme of 32 plant genera. Brazilian Journal of Genetics, Ribeirão Preto, v.18. n.3, p.441-502, 1995.

BORDIGNON, R. Características agronômicas e tolerância à triste$z a$ de híbridos entre porta-enxertos elite de citros. 2000. 164p. Tese (Doutorado em Genética e Biologia Molecular) - Instituto de Biologia, Universidade Estadual de Campinas.

BORDIGNON, R. Hibridações interespecíficas, intergenéricas, intergrupais, intersubtribais e intersubfamiliares de Citrus e gêneros relacionados. 1995. 104f. Tese (Mestrado em Genética) - Instituto de Biologia, Universidade Estadual de Campinas.

BORDIGNON, R.; MEDINA FILHO, H.P.; SIQUEIRA, W.J.; Genetics of tolerance to the tristeza disease in citrus rootstock. Genetics and Molecular Biology, Ribeirão Preto, v.27, n.1, 2004. (no prelo)

BORDIGNON, R.; MEDINA FILHO, H.P.; BALLVÉ, R.M.L. Melhoramento genético de citros no Instituto Agronômico. Laranja, Cordeirópolis, v.11, p.167-176, 1990.

BORDIGNON, R.; MEDINA FILHO, H.P.;SIQUEIRA, W.J.; PIO, R.M.; J. Efeito da tristeza em características vegetativas, produtivas e industriais da laranja 'Valência' enxertada em portaenxertos híbridos segregando para tolerância. Bragantia, Campinas, v.62, n.2, p.207-215, 2003.
CAMERON, J.W.; FROST, H.B. Genetics, breeding and nucellar embriony. In: REUTHER, W; BARTCHERLOR, L.D.; WEBBER, H.J. (Eds). The Citrus Industry. Berkeley: University of California Press, 1968. v.II, p. 325-370.

CASTLE, W.S. Citrus rootstocks. In: ROM, R.C.; CARLSON, R.F. (Eds). Rootstocks for Fruit Crops. New York: J. Wiley and Sons, 1987. p. 361-399.

CASTLE, W.S.; TUCKER, D.P.H.; KREZDORN, A.H.; YOUTSEY, C.O. Rootstocks selection: the first step to success. In: WOESTE, J.T. (Ed). Rootstocks for Florida citrus. Gainesville: University of Florida, 1989. 47p.

CLEVELAND, W.S. Robust locally weighted and smoothing scatter plots. Journal of the American Statistical Association, Alexandria, v.74, p.829-836, 1979.

DI GIORGI, F.; YDE, B.Y.; TRIBONI, H.R.; MARCHI, R.J.; WAGNER, R.L. Qualidade da laranja para industrialização. Laranja, Cordeirópolis, v.14, n.1, p.97-118, 1993.

DIEZ, J.C.; MÜLLER, I.A. Performance preliminar de laranjeira Valência enxertada sobre 44 porta-enxertos. In: SEMINÁRIO INTERNACIONAL DE CITROS, I., 1990, Bebedouro. Anais... Jaboticabal: FUNEP, 1990. p.123-133.

DOMINGUES, E.T.; TEÓFILOSOBRINHO, J.;MATTOS JÚNIOR, D.; POMPEU JÚNIOR, J.; OLIVEIRA, L.A. Qualidade e maturação das laranjas Ovale, Ovale de Siracusa e Ovale San Lio. Laranja, Cordeirópolis, v.17, n.1, p.143-158, 1996.

DUARTE, J.B. Sobre o emprego e a análise estatística do delineamento em blocos aumentados no melhoramento genético vegetal. 2000. 293f. Tese (Doutorado)- Escola Superior de Agricultura "Luis de Queiroz", Universidade de São Paulo, Piracicaba.

FERGUSON, L.; SAKOVICH, N.; ROOSE, M. California Citrus Rootstocks. Riverside: University of California, Division of Agriculture and Natural Resources, 1990.18p. (Publication 21477)

FIGUEIREDO, J.O.; POMPEU JÚNIOR, J.; PIO, R.M.; TEÓFILO SOBRINHO, J.; DOMINGUES, E.T.; LARANJEIRA, F.F. Produção inicial do tangor Murcote sobre dezesseis porta-enxertos em São Paulo. Laranja, Cordeirópolis, v.18, n.1, p.165-173, 1997.

MÜLLER, G.W.; TEÓFILO SOBRINHO, J.; DOMINGUES, E.T. Compatibilidade da laranja Pêra clone Bianchi sobre doze porta-enxertos após 23 anos de plantio. Laranja, Cordeirópolis, v.17, n.1, p.123-141, 1996.

PHILLIPS, R.L. Dwarfing rootstocks for citrus. In: INTERNATIONAL CITRUS SYMPOSIUM. 1968, Riverside. Proceedings... Riverside: International Society of Citriculture, 1969. v.1, p.401-406.

POMPEU JÚNIOR, J. Porta-enxertos. In: RODRIGUES, O.; VIEGAS, F.; POMPEU JÚNIOR, J.; AMARO, A.A. (Eds). Citricultura Brasileira, 2.ed. Campinas: Fundação Cargil, 1991. v.1, p.265-280.

POMPEU JÚNIOR, J. Situação do uso de porta-enxertos no Brasil. In: SEMINÁRIO INTERNACIONAL DE CITROS. I., 1990, Bebedouro. Anais... Jaboticabal, FUNEP, 1990. p.1-10. 
ROOSE, M.L.; COLE, D.A.; ATKIN, D.; KUPPER, R.S. Yield and tree size of four citrus cultivars on 21 rootstocks in California. Journal of the American Society for Horticultural Science, Alexandria, v.114, n.4, p.678-684, 1989.

STUCHI, E.S.; SEMPIONATO, O.R.; SILVA, J.A.A. Influência dos porta-enxertos na qualidade dos frutos cítricos. Laranja, Cordeirópolis, v.17, n.1, p.159-178, 1996.

TEÓFILO SOBRINHO, J. Comportamento da laranjeira Valência (Citrus sinensis L., Osbeck) sobre diferentes porta-enxertos. 1972. Dissertação (Doutorado) - Escola Superior de Agronomia "Luiz de Queiroz", Universidade de São Paulo, Piracicaba.

TEÓFILO SOBRINHO, J.; POMPEU JÚNIOR, J.; FIGUEIREDO, J.O.; MÜLLER, G.W.; LARANJEIRA, F.F.; DOMINGUES, E.T. Influência de onze porta-enxertos na produção e qualidade dos frutos da laranjeira Pêra clone Bianchi. Laranja, Cordeirópolis, v.20, n.1, p.153-166, 1999.
VIEIRA, S.R. Geoestatística em estudos de variabilidade espacial do solo. In: NOVAIS, R.F.; ALVAREZ, V.V.H.; SCHAEFER, C.E.G.R. (Eds). Tópicos em Ciência do Solo. Viçosa: Sociedade Brasileira de Ciência do Solo, 2000. v.1, p.1-54.

WEBBER, H.J. Rootstocks: their character and reactions. In: WEBBER, H.J.; BATCHERLOR, L.D. (Eds). The Citrus Industry. Berkeley: University of California Press. 1948, v.II, p.69-168. 